\title{
Gas-phase and heat-exchange effects on the ignition of high- and low-exothermicity porous solids subject to constant heating
}

\author{
A. A. Shah • J. Brindley • A. C. McIntosh • \\ J. Griffiths
}

Received: 1 December 2004 / Accepted: 12 April 2006 / Published online: 18 November 2006

(C) Springer Science+Business Media B.V. 2006

\begin{abstract}
This article investigates the ignition of low-exothermicity reactive porous solids exposed to a maintained source of heat (hotspot), without oxygen limitation. The gas flow within the solid, particularly in response to pressure gradients (Darcy's law), is accounted for. Numerical experiments related to the ignition of low-exothermicity porous materials are presented. Gas and solid products of reaction are included. The first stage of the paper examines the (pseudo-homogeneous) assumption of a single temperature for both phases, amounting to an infinite rate of heat exchange between the two. Isolating the effect of gas production and flow in this manner, the effect of each on the ignition time is studied. In such cases, ignition is conveniently defined by the birth of a self-sustained combustion wave. It is found that gas production decreases the ignition time, compared to equivalent systems in which the gas-dynamic problem is effectively neglected. The reason for this is quite simple; the smaller heat capacity of the gas allows the overall temperature to attain a higher value in a similar time, and so speeds up the ignition process. Next, numerical results using a two-temperature (heterogeneous) model, allowing for local heat exchange between the phases, are presented. The pseudo-homogeneous results are recovered in the limit of infinite heat exchange. For a finite value of heat exchange, the ignition time is lower when compared to the single-temperature limit, decreasing as the rate of heat exchange decreases. However, the decrease is only mild, of the order of a few percent, indicating that the pseudo-homogeneous model is in fact a rather
\end{abstract}

\footnotetext{
A. A. Shah $(\varangle) \cdot$ J. Brindley

Applied Mathematics Department, Leeds University, Leeds LS29JT, UK

e-mail: ashah@pims.math.ca
}

Present Address:

A. A. Shah

Department of Mathematics,

Simon Fraser University, 8888 University Drive,

B.C. Canada V5A 1S6

A. C. McIntosh

SPEME, Leeds University, Leeds LS29JT, UK

J. Griffiths

School of Chemistry, Leeds University,

Leeds LS29JT, UK 
good approximation, at least for a constant heat-exchange rate. The relationships between the ignition time and number of physico-chemical parameters of the system are also investigated.

Keywords Combustion wave $\cdot$ Heat exchange $\cdot$ Hotspot $\cdot$ Ignition $\cdot$ Low-exothermicity

\section{Introduction}

The ignition of highly exothermic porous solids, such as explosives and propellants, is an issue of great practical importance, particularly when materials of this nature are inadvertently (or otherwise) exposed to thermal energy from a heat source (hotspot). In the agriculture and shipping industries, amongst others, an equally important issue is the exposure of low-exothermicity particulate or fibrous solids to heat sources such as overheated bearings in grain-handling equipment, pilot lights or electrical arcs. In such cases the hotspot invariably causes some destruction through slow local combustion, and perhaps, more importantly, it may initiate a self-propagating combustion wave, which implies total loss of the material and could possibly lead to some greater catastrophe such as fire or explosion. Clearly, in both cases, an understanding of the conditions under which exothermic reaction occurs, the extent of decomposition and predictions of ignition times are crucial for hazard prevention.

Liñan and Williams [1], and Kapila [2], have developed an asymptotic theory for the ignition of a highly exothermic semi-infinite slab subjected to a maintained heat flux at one end. These studies provide a leading-order prediction of the ignition time, which is defined as the time taken to reach some critical temperature at which the reaction rate becomes significant. Because of the large heat release, reactant consumption in these studies can be neglected in the time leading up to ignition, which always takes place at the hotspot surface. The effects of reactant consumption, a non-planar geometry and gas-dynamics introduce significant complications. Telengator and co-authors, [3, 4], have recently performed an analysis of hotspot ignition in a one-dimensional porous medium model, by making the same assumption of negligible reactant-consumption. However they did not include any pressure-driven flow effects and neglected the hindrance to the flow caused by the presence of the porous solid. In this work we shall include such effects.

For a constant hotspot heat-flux, ignition criteria associated with low-exothermicity solids have been obtained numerically in [5-9] for the finite slab, cylinder and sphere. In contrast to high-exothermicity solids, the calculations reveal that:

(i) a substantial degree of reactant consumption occurs before the onset of ignition;

(ii) ignition typically occurs away from the hotspot boundary, within the virgin solid;

(iii) reactant consumption can prevent the formation of a combustion wave.

In fact, because of (iii), a more natural definition of 'ignition' is that a self-sustained combustion wave is initiated, and this is one which we shall adopt in the latter sections of this paper. This of course implies that the ignition (or critical) temperature at which a wave would be guaranteed in high-exothermicity materials has no precise analogue for low-exothermicity materials.

Because of the many similarities, it is also worth drawing attention to a number of papers related to filtration combustion (FC) waves, typically involving a heterogeneous exothermic reaction front propagating through a porous solid which reacts with a gas carrying oxidizer flowing through its pores. Other related applications of FC include important processes such as smouldering and self-propagating high-temperature synthesis (see [10] and [11] for a review). Although much of the literature is concerned with the propagation of such waves, initiation problems have also received attention, [12-14]. In FC, gas is assumed to be forced through the material, effectively leading to a constant temperature at one end. Instead we shall assume a constant heat flux, and that any convection is engendered by pressure gradients arising first from the hotspot heating, and then from any associated combustion, rather than through any forcing. 
The outline of this paper is as follows. In Sect. 2 we derive the mathematical model which will be employed in the next two sections. This model assumes local temperature equilibrium between the solid and gas phases and will be called the Pseudo-Homogeneous (PH) model. In Sect. 3 we present numerical calculations associated with the burning of low- and $O(1)$-exothermicity finite slabs, using the PH model. We demonstrate the effect that the gas phase has on ignition (defined as the birth of a self-sustained combustion wave), particularly when a substantial volume of gas is created during reaction. In Sect. 4 we move to a mathematical model which employs separate energy (temperature) equations for the two phases. We call this the Heterogeneous model and label it $\mathrm{H}$. Numerical calculations reveal further information regarding the effect of the gas phase on ignition, now with the effect of heat exchange. We further demonstrate the effects on the ignition time of changes in a number of important physico-chemical parameters of the system. Finally, in Sect. 5 we discuss our results and outline current and future work, particularly the effect that oxygen dependence and/or restriction would have on our results.

\section{Model and equations for a single temperature}

Consider a rigid porous material, $X$, initially permeated by air, which undergoes an exothermic reaction to form gaseous and solid products ( $Z$ and $S$, respectively):

$X \rightarrow a S+b Z$,

where $a$ and $b$ are the stoichiometric coefficients. The mathematical description of this process will involve both the reaction-diffusion and gas-dynamic representations. Before presenting the model, we list the assumptions and some explanatory comments.

(1) We assume that the oxygen supply is always adequate locally to support combustion - thus the reaction is not oxygen-limited. The oxygen may be provided by transport of air through the pores, for high-exothermicity materials, or alternatively it may be provided by the primary decomposition of the solid.

(2) We assume a single temperature for both gas and solid, i.e., local temperature equilibrium between the two phases. Although this assumption is widely used, see for example [3, 4, 10, 12-14, 15-18] and references therein, we stress that one must generally allow for finite rates of inter-phase heat transfer. For this reason we shall drop this assumption in Sect. 4. The results of that section will allow us to draw conclusions about the effectiveness of the PH model.

(3) As in $[3,4,10-14,15-18]$ we neglect structural defects of the porous solid in our model. This allows us to focus on the effects of the gas movement, gas production and inter-phase heat transfer that leads up to the ignition event, after which structural deformation could occur. For details of the effects of cracking and deformation the reader is referred to [19-21].

(4) Since, the reacting-solid conservation equation does not contain diffusive or convective-transport terms, we need only a gas continuity equation, which relates the gas density and velocity to the porosity in each control volume. This equation will also contain a source term from that fraction of the mass of $X$ which forms $Z$.

(5) We assume a single density, specific heat capacity and thermal conductivity for the gas phase, $Z+$ air, and similarly for the solid phase, $X+S$. The subscript $g(s)$ refers to the common value of a gas (solid) phase property.

(6) For the motion of the gas through the pores of the solid we assume Darcy's law and the ideal-gas law.

(7) In any control volume it is necessary to specify how much of the products of reaction are gas, $Z$, and solid, $S$, as fractions of the mass of $X$ which has reacted. Let $V^{\prime}$ be a control volume in which the porosity is given by $\phi=\phi(x, t)$ and the volume fraction of solid reactant, $X$, is given by $\psi=\psi(x, t)$, at any time $t$ and location $x$ in $V^{\prime}$. The volume fraction vacated by any reacted $X$, from the initial time, is given by $\left(1-\phi_{0}\right)-\psi$, where $\phi_{0}$ is the initial porosity. Thus, the mass of the reacted $X$ is given by 
$m_{X}^{\prime}=\left(1-\phi_{0}-\psi\right) V^{\prime} \rho_{\mathrm{s}}^{\prime}$, where $\rho_{\mathrm{s}}^{\prime}$ is the (constant) density of $X$. The mass of solid product must then be $m_{S}^{\prime}=\epsilon m_{X^{\prime}}$, where $\epsilon$ is the fraction of the mass of $X$ which forms $S$. Since we are assuming that the solid product has the same density as $X$, the volume occupied by $S$ is given by $V_{S^{\prime}}=m_{S}^{\prime} / \rho_{\mathrm{s}}^{\prime}=\epsilon\left(1-\phi_{0}-\psi\right) V^{\prime}$, and therefore the volume fraction of $S$, at any time $t$ and location $x$ in $V^{\prime}$, is $\epsilon\left(1-\phi_{0}-\psi(x, t)\right)$. From the fact that the sum of all volume fractions must be unity, we finally obtain:

$\psi=1-\frac{\phi-\epsilon \phi_{0}}{1-\epsilon}$

From this relationship, we can conveniently eliminate the solid concentration as a dependent variable in favour of the volume fraction, $\psi$, of $X$.

Based on the preceding comments, we can write the equations for energy (temperature), gas continuity, the ideal-gas law and Darcy's law as follows:

$\frac{\partial}{\partial t^{\prime}}\left(\left(\rho C_{\mathrm{p}}\right)^{\prime} T^{\prime}\right)+C_{\mathrm{g}}^{\prime} \frac{\partial}{\partial x^{\prime}}\left(\rho_{\mathrm{g}}^{\prime} u_{\mathrm{g}}^{\prime} \phi T^{\prime}\right)-\frac{\partial}{\partial x^{\prime}}\left(\lambda^{\prime} \frac{\partial T^{\prime}}{\partial x^{\prime}}\right)=-Q^{\prime} \rho_{\mathrm{s}}^{\prime} \frac{\partial \psi}{\partial t^{\prime}}$,

$\frac{\partial}{\partial t^{\prime}}\left(\rho_{\mathrm{g}}^{\prime} \phi\right)+\frac{\partial}{\partial x^{\prime}}\left(\rho_{\mathrm{g}}^{\prime} u_{\mathrm{g}}^{\prime} \phi\right)=-(1-\epsilon) \rho_{\mathrm{s}}^{\prime} \frac{\partial \psi}{\partial t^{\prime}}$,

$P_{\mathrm{g}}^{\prime}=\frac{\rho_{\mathrm{g}}^{\prime} R^{\prime} T^{\prime}}{W_{\mathrm{g}}^{\prime}}$

$u_{\mathrm{g}}^{\prime}=-\frac{\kappa^{\prime}}{v^{\prime}} \frac{\partial P_{\mathrm{g}}^{\prime}}{\partial x^{\prime}}$

where

$\lambda^{\prime} \equiv \lambda_{\mathrm{s}}^{\prime}(1-\phi)+\lambda_{\mathrm{g}}^{\prime} \phi$

$\left(\rho C_{\mathrm{p}}\right)^{\prime} \equiv \rho_{\mathrm{s}}^{\prime} C_{\mathrm{s}}^{\prime}(1-\phi)+\rho_{\mathrm{g}}^{\prime} C_{\mathrm{g}}^{\prime} \phi$,

$-\frac{\partial \psi}{\partial t^{\prime}}=\Omega^{\prime} \equiv A^{\prime} \psi \mathrm{e}^{-E^{\prime} / R^{\prime} T^{\prime}}$

$\psi=1-\frac{\phi-\epsilon \phi_{0}}{1-\epsilon}$.

In these equations $T$ is the (single) temperature, $P_{\mathrm{g}}^{\prime}$ is pressure; $\rho_{\mathrm{g}}^{\prime}\left(\rho_{\mathrm{s}}^{\prime}\right), \lambda_{\mathrm{g}}^{\prime}\left(\lambda_{\mathrm{s}}^{\prime}\right)$ and $C_{\mathrm{g}}^{\prime}\left(C_{\mathrm{s}}^{\prime}\right)$ are the (actual) density, thermal conductivity and specific heat capacity of the gas (solid) phase; $\Omega^{\prime}$ is the Arrhenius reaction rate, with pre-exponential $A^{\prime}$ and activation energy $E^{\prime}, W_{\mathrm{g}}^{\prime}$ is the molar mass of the gas phase, $Q^{\prime}$ is the heat release per $\mathrm{kg}$ of $X, \kappa^{\prime}$ is the permeability of the solid phase, $v^{\prime}$ is the dynamic viscosity of the gas, and $u_{\mathrm{g}}^{\prime}$ is the gas velocity.

The boundary conditions for temperature are:

$T^{\prime}\left(x^{\prime}=x_{1}^{\prime}, t^{\prime}\right)=T_{0}^{\prime}=$ const.,$\quad-\lambda^{\prime} \frac{\partial T^{\prime}}{\partial x^{\prime}}\left(0, t^{\prime}\right)=p^{\prime}$,

corresponding to a total heat flux $p^{\prime}$ applied at $x^{\prime}=0$, and ambient conditions at the outer edge of the solid, $x^{\prime}=x_{1}^{\prime}$. For the gas density we have:

$\rho_{\mathrm{g}}^{\prime}\left(x^{\prime}=x_{1}^{\prime}, t^{\prime}\right)=\rho_{\mathrm{g} 0}=$ const.

The conditions at the outer edge are consistent with atmospheric pressure, $P_{\text {at }}^{\prime}$, and the gas velocity is zero at the hotspot surface:

$P^{\prime}\left(x^{\prime}=x_{1}^{\prime}, t^{\prime}\right)=P_{\mathrm{at}}^{\prime}, \quad u_{\mathrm{g}}^{\prime}\left(0, t^{\prime}\right)=0$. 
Finally, the initial conditions are given by:

$T^{\prime}\left(x^{\prime}, 0\right)=T_{0}^{\prime}, \quad \rho_{\mathrm{g}}^{\prime}\left(x^{\prime}, 0\right)=\rho_{\mathrm{g} 0}^{\prime}, \quad \phi\left(x^{\prime}, 0\right)=1-\psi\left(x^{\prime}, 0\right)=\phi_{0}$.

In Darcy's law, (2d), we employ the semi-empirical Kozeny-Carman (KC) law to define $\kappa^{\prime}$ (see [22]):

$\frac{\kappa^{\prime}}{v^{\prime}}=\frac{d_{\mathrm{p}}^{\prime 2}}{k_{\mathrm{KC}}^{\prime}{ }^{2} \tau^{\prime} v^{\prime}} \frac{\phi^{3}}{(1-\phi)^{2}} \equiv k^{\prime} \frac{\phi^{3}}{(1-\phi)^{2}}$,

where $d_{\mathrm{p}}^{\prime}$ is an average pore size, $k_{\mathrm{KC}}^{\prime}$ is a constant referred to as the $\mathrm{KC}$ shape-factor and $k^{\prime}$ is called the $\mathrm{KC}$ constant.

We assume that gas diffusion satisfies Fick's law, which will be true provided that the Knudsen number $\left(=d_{\mathrm{mf}}^{\prime} / d_{\mathrm{p}}^{\prime}\right.$, where $d_{\mathrm{mf}}^{\prime}$ is the mean-free path of the gas) remains smaller than unity. This condition is also required for the $\mathrm{KC}$ law to hold. We also point out that the 'effective' diffusion coefficient of the gas is found from the molecular diffusion coefficient, by taking into account the porosity and tortuosity of the solid (see [23] for more details). With a tortuosity of around 1-4, for porosities in the range $0 \cdot 1-0 \cdot 6$, the molecular-diffusion coefficient is reduced by a factor of between $1 / 40$ and $1 / 2$. Although this is not directly relevant to the present paper, it will play a crucial role in the combustion of systems in which input of oxygen is required and/or restricted; this is the subject of a forthcoming paper.

\subsection{Non-dimensionalization}

We non-dimensionalize Eqs. 2-6 with the following scales:

$$
\begin{gathered}
T=\frac{T^{\prime}}{T_{0}^{\prime}}, \quad \rho_{\mathrm{g}}=\frac{\rho_{\mathrm{g}}^{\prime}}{\rho_{\mathrm{g} 0}^{\prime}}, \quad P=\frac{P^{\prime}}{P_{\mathrm{at}}^{\prime}}, \quad u_{\mathrm{g}}=\frac{\rho_{\mathrm{s}}^{\prime} C_{\mathrm{s}}^{\prime} T_{0}^{\prime} u_{\mathrm{g}}^{\prime}}{p^{\prime}}, \quad x=\frac{p^{\prime} x^{\prime}}{\lambda_{\mathrm{s}}^{\prime} T_{0}^{\prime}}, \quad E=\frac{E^{\prime}}{R^{\prime} T_{0}^{\prime}}, \\
t=\frac{p^{\prime 2} t^{\prime}}{\lambda_{\mathrm{s}}^{\prime} \rho_{\mathrm{s}}^{\prime} C_{\mathrm{s}}^{\prime} T_{0}^{\prime 2}}, \quad A=\frac{\lambda_{\mathrm{s}}^{\prime} \rho_{\mathrm{s}}^{\prime} C_{\mathrm{s}}^{\prime} T_{0}^{\prime 2} A^{\prime}}{p^{\prime 2}}, \quad Q=\frac{Q^{\prime}}{C_{\mathrm{s}}^{\prime} T_{0}^{\prime}}, \quad k=\frac{\rho_{\mathrm{s}}^{\prime} C_{\mathrm{s}}^{\prime} P_{\mathrm{at}}^{\prime} k^{\prime}}{\lambda_{\mathrm{s}}^{\prime}}, \\
\alpha=\frac{C_{\mathrm{g}}^{\prime}}{C_{\mathrm{s}}^{\prime}}, \quad \delta=\frac{\rho_{\mathrm{g}}^{\prime}}{\rho_{\mathrm{s}}^{\prime}}, \quad \gamma=\frac{\lambda_{\mathrm{g}}^{\prime}}{\lambda_{\mathrm{s}}^{\prime}}, \quad x_{1}=\frac{p^{\prime} x_{1}^{\prime}}{\lambda_{\mathrm{s}}^{\prime} T_{0}^{\prime}},
\end{gathered}
$$

yielding:

$\frac{\partial}{\partial t}\left((1-\phi) T+\alpha \delta \rho_{\mathrm{g}} \phi T\right)+\alpha \delta \frac{\partial}{\partial x}\left(\rho_{\mathrm{g}} u_{\mathrm{g}} \phi T\right)-\frac{\partial}{\partial x}\left((1-\phi+\gamma \phi) \frac{\partial T}{\partial x}\right)=-Q \frac{\partial \psi}{\partial t}$,

$\frac{\partial}{\partial t}\left(\rho_{\mathrm{g}} \phi\right)+\frac{\partial}{\partial x}\left(\rho_{\mathrm{g}} u_{\mathrm{g}} \phi\right)=-(1-\epsilon) \delta^{-1} \frac{\partial \psi}{\partial t}$,

$u_{\mathrm{g}}=-k \frac{\phi^{3}}{(1-\phi)^{2}} \frac{\partial P_{\mathrm{g}}}{\partial x}$,

$P_{\mathrm{g}}=\rho_{\mathrm{g}} T$,

$-\frac{\partial \psi}{\partial t}=\Omega \equiv A \psi \mathrm{e}^{-E / T}$ 
$\psi=1-\frac{\phi-\epsilon \phi_{0}}{1-\epsilon}$.

The boundary conditions, (4) and (5), become:

$T\left(x_{1}, t\right)=1, \quad(1-\phi+\gamma \phi) \frac{\partial T}{\partial x}(0, t)=-1$,

$\rho_{\mathrm{g}}\left(x_{1}, t\right)=1, \quad u_{\mathrm{g}}(0, t)=0$,

and the initial conditions, (6), are now:

$T(x, 0)=1, \quad \rho_{\mathrm{g}}(x, 0)=1, \quad \phi(x, 0)=1-\psi(x, 0)=\phi_{0}, \quad u_{\mathrm{g}}(x, 0)=0$.

\section{Pseudo-homogeneous results: PH model}

Crucial to the result of the high-exothermicity analyses in, for example, [3, 4], is that reactant consumption is negligible in the time leading up to ignition, defined to be when the temperature at the hotspot surface reaches a critical value, $T_{\mathrm{c}}$. In low-exothermicity materials a significant degree of reactant consumption takes place before any critical temperature is reached, [5-9], and, in contrast to the high-exothermicity case, the realization of a critical (so-called ignition) temperature is not a guarantee of a combustion wave. It is natural therefore to re-define 'ignition' for low-exothermicity materials to mean that a self-sustained combustion wave is formed, i.e., that the wave continues to the boundary, even if the hotspot heating is switched off after it has formed.

In this section, we present some numerical computations relating to the ignition of porous solids with low and $O(1)$ values of $Q$. We use the PH model, (8)-(10), although we switch to the Heterogeneous model in the next section. In both sections, we consider the following questions: what is the effect on the ignition time (as defined above) of:

(i) gas production and movement?

(ii) heat exchange between the two phases?

(iii) the properties of the gas and solid phases?

Question (i) is partly answered in this section by isolating the gas-dynamic problem from that of heat exchange. Answers to the remaining two questions and a fuller answer to the first will follow in the next section.

Unless otherwise specified, the parameter values employed in this section are given in Table 1. In particular, the values of $Q^{\prime}, C_{\mathrm{p}}^{\prime}$ and $T_{0}^{\prime}$ yield $Q=1.11$ (see definition (7) and earlier discussions). Note that we replace the infinite domain of the previous section with a finite one of length $x_{1}^{\prime}$ (dimensionless length $x_{1}$ ). The numerical strategy is detailed in the appendix, for both the $\mathrm{PH}$ and $\mathrm{H}$ models.

We first note that for values of power, $p^{\prime}$, greater than a unique critical value, $p_{c}^{\prime}$, there is found to be a transition from slow-burning to quick-burning behaviour, in which a self-sustained combustion wave is formed. Thus, there is no ambiguity in our definition of criticality. We shall always use the value $p^{\prime}=1000$ $\mathrm{W} \mathrm{m}^{-2}$, which gives a combustion wave in all cases considered. Note that in previous work concentrating solely on reaction and diffusion, [5, 8], it was found that the critical power was unique for both the slab and cylindrically symmetric geometries. In contrast, for a spherically symmetric geometry it was found that for small-radii hotspots three distinct critical powers exist, $p_{1}^{\prime}<p_{2}^{\prime}<p_{3}^{\prime}$. For $p<p_{1}^{\prime}$ no wave is formed; for $p_{1}^{\prime}<p^{\prime}<p_{2}^{\prime}$ a wave is formed; for $p_{2}^{\prime}<p^{\prime}<p_{3}^{\prime}$ no wave is formed; and for $p>p_{3}^{\prime}$ a wave is formed. Evidence of such behaviour was investigated in the present case but was not found to exist. The interesting question of whether such behaviour can be found for a spherically symmetric analogue of the present system is left as future work.

Let us define an 'approximate ignition time', $\tau_{1 / 2}$, as the time taken for the reactant volume fraction at $x=0, \psi(0, t)$, to reach half of its initial value, $\left(1-\phi_{0}\right) / 2$, with $p^{\prime}=1000 \mathrm{~W} \mathrm{~m}^{-2}$. This measure is reasonably 
Table 1 Typical parameter values. These values are intended to be representative of some low-exothermicity materials in small-scale tests in laboratory experiments, [24]

\begin{tabular}{lll}
\hline Quantity & Symbol & Value \\
\hline Pre-exponential factor & $A^{\prime}$ & $10^{5} \mathrm{~s}^{-1}$ \\
Heat of reaction & $Q^{\prime}$ & $4 \times 10^{5} \mathrm{~J} \mathrm{~kg}^{-1}$ \\
Activation energy & $E^{\prime}$ & $6 \cdot 8 \times 10^{7} \mathrm{~J} \mathrm{kmol}^{-1}$ \\
KC constant & $k^{\prime}$ & $1 \times 10^{-7} \mathrm{~kg} \mathrm{~m}^{2} \mathrm{~s}$ \\
Solid density & $\rho_{\mathrm{s}}^{\prime}$ & $350 \mathrm{~kg} \mathrm{~m}^{-3}$ \\
Specific heat of solid & $C_{\mathrm{s}}^{\prime}$ & $1200 \mathrm{~J} \mathrm{~kg}^{-1} \mathrm{~K}^{-1}$ \\
Specific heat of gas & $C_{\mathrm{g}}^{\prime}$ & $1000 \mathrm{~J} \mathrm{~kg}^{-1} \mathrm{~K}^{-1}$ \\
Thermal conductivity of solid & $\lambda_{\mathrm{s}}^{\prime}$ & $0 \cdot 4 \mathrm{~W} \mathrm{~m}^{-1} \mathrm{~K}^{-1}$ \\
Thermal conductivity of gas & $\lambda_{\mathrm{g}}^{\prime}$ & $0 \cdot 1 \mathrm{~W} \mathrm{~m}^{-1} \mathrm{~K}^{-1}$ \\
Ambient/initial temperature & $T_{0}^{\prime}$ & $300 \mathrm{~K}$ \\
Initial oxygen density & $\rho_{g 0}^{\prime}$ & $1.5235 \mathrm{~kg} \mathrm{~m}^{-3}$ \\
Molar mass of gas & $W_{\mathrm{g}}^{\prime}$ & $0 \cdot 038 \mathrm{~kg} \mathrm{~mol}^{-1}$ \\
Applied heat flux & $p^{\prime}$ & $1000 \mathrm{~W} \mathrm{~m} \mathrm{~W}^{-2}$ \\
Initial porosity & $\phi_{0}$ & $0 \cdot 3$ \\
Length of sample & $x_{1}^{\prime}$ & $0 \cdot 1 \mathrm{~m}$ \\
Fraction of reacted mass forming solid $S$ & $\epsilon^{\prime}$ & $0 \cdot 8$ \\
Heat-exchange parameter & $\mu^{\prime}$ & $50 \mathrm{~J} \mathrm{~m}^{-3} \mathrm{~K}^{-1} \mathrm{~s}^{-1}$ \\
\hline
\end{tabular}

accurate because in all cases considered a combustion wave was formed relatively soon after $\psi(0, t)$ reaches $\left(1-\phi_{0}\right) / 2$ and this combustion wave propagated independently of the hotspot heating, i.e., if the heating was switched off, the wave would continue to the boundary. Based on a typical set of results, we provide more justification later (we point out that this definition is designed to approximate the time at which a self-propagating wave is formed but that alternative definitions would apply. For example, the time at which the evolution of $\psi(x, t)$ at $x=0$ has the greatest slope).

With this definition we note that:

- $\tau_{1 / 2}$ is almost insensitive to the value of $k^{\prime}$, with only very mild reductions as $k^{\prime}$ is decreased (less than $1 \%$ ), at fixed values of the other parameters.

- We can compare $\tau_{1 / 2}$ to the value for an equivalent system in which the gas flow is neglected, no gas is formed during reaction and the density of the gas remains constant. In such systems, the density and thermal conductivity are essentially those of the bulk material (they are averaged with respect to the volume fractions of the gas and solid). The specific heat capacity, not being measured by volume, has to be treated with more care, and is calculated from the volume-averaged heat capacity (not specific heat capacity) divided by the bulk density. Equations 8-10 reduce to a reaction-diffusion set. Using the values given in Table 1, Fig. 1 demonstrates the evolution of temperature and reactant volume fraction when gas flow is neglected in this manner. Clearly, the hotspot heating at $x=0$ generates a self-propagating combustion wave that leads to complete depletion of the reactant. Waves of this type have been discussed at length in [5-9], as mentioned earlier.

Again using the values of Table 1, the PH system, (8)-(10), yields the results shown in Figs. 2 and 3. The information we seek is contained in Fig. 2, which shows the evolution of temperature and porosity through the ignition stage and into the birth of a self-propagating wave. Comparing the profiles in Fig. 2 with those in Fig. 1, we see that the ignition time, indicated by the comparatively rapid development of high temperature and porosity gradients and measured by $\tau_{1 / 2}$, is about $20-30 \mathrm{~s}$ shorter. We can also see that, once the combustion wave has passed, its speed of propagation is significantly increased by the movement of the hot gas through the unreacted porous solid, which adds to the preheating effect produced by conduction. This is true for all $k^{\prime}>0$ and the wave speed increases with increasing $k^{\prime}$.

We now refer to Fig. 4, which shows the value of $\tau_{1 / 2}$ as $\epsilon$, the fraction of mass that forms solid, is decreased, for three values of the initial porosity, $\phi_{0}$. The systems in which gas flow is neglected, as 

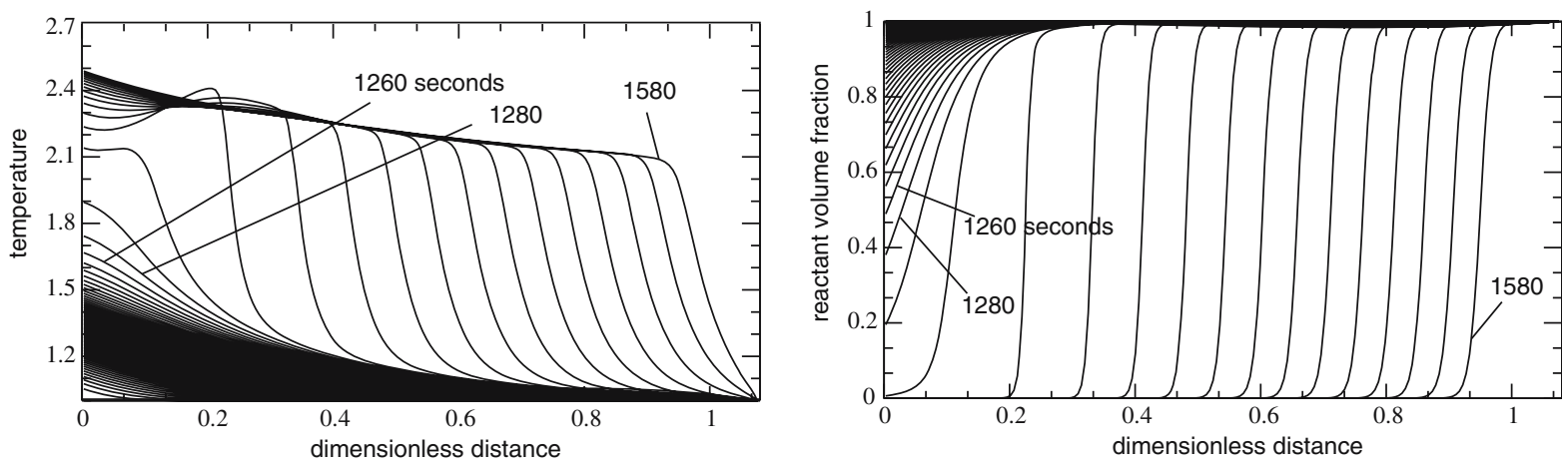

Fig. 1 Profiles of temperature and reactant volume fraction when gas flow is neglected, based on the values in Table 1. Compare the time of ignition with those of (i) the PH model, Fig. 2; and (ii) the H model, Fig. 6.
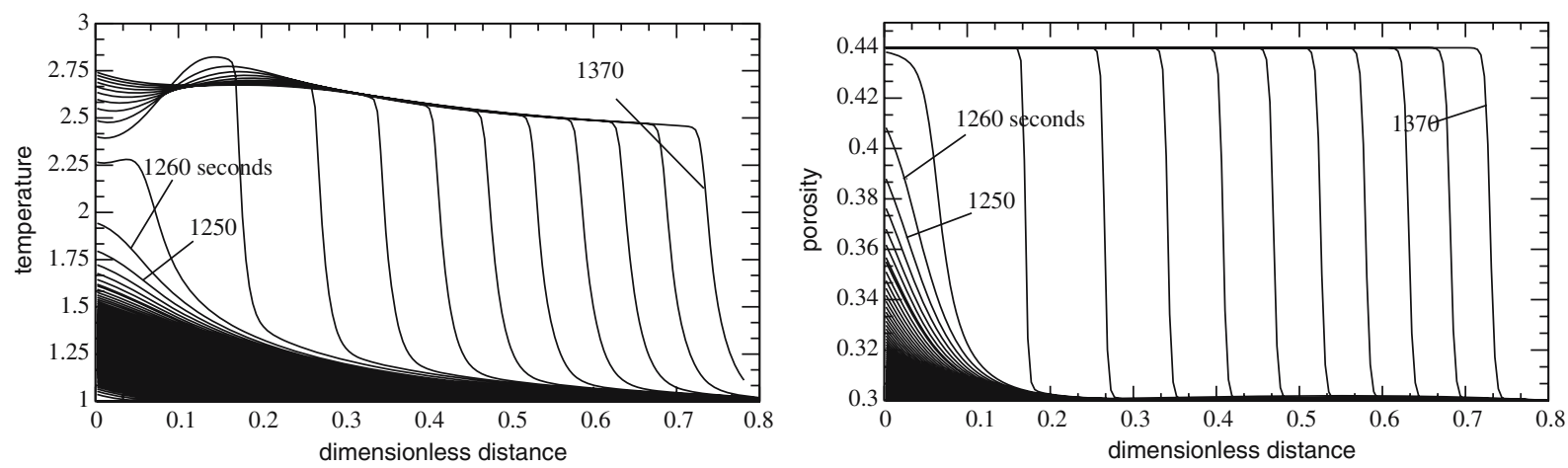

Fig. 2 Temperature and porosity profiles for the $\mathrm{PH}$ example corresponding to the values in Table 1. Compare with those of (i) no gas movement, Fig. 1; and (ii) the H model, Fig. 6

described above, correspond to the axis $\epsilon=1$. As we would expect, $\tau_{1 / 2}$ decreases as $\phi_{0}$ decreases, reflecting the fact that less reactant is required to burn. We also find that the more gas is produced ( $\epsilon$ is decreased), the shorter the value of $\tau_{1 / 2}$. This is a direct result of the smaller heat capacity of the gas compared with the solid, and therefore the larger averaged temperature during the ignition stage; hence the discrepancy in ignition times in Figs. 1 and 2. There is, however, a sensitive balance in specific heat capacity. If we gradually increase $C_{\mathrm{g}}^{\prime}$, as shown in Fig. 5, we eventually find the reverse trend, i.e., ignition time increasing as $\epsilon$ decreases. For a two-temperature model, we shall find a slightly different behaviour.

These conclusions are of course only valid if we assume that the gas and solid temperatures equilibrate rapidly enough for the difference to be neglected. In most systems the rate of heat exchange will have a finite value, which will inevitably affect these results. We now demonstrate this effect both on the ignition time and the combustion-wave speed.

\section{Heterogeneous results: $\mathrm{H}$ model}

For the $\mathrm{H}$ calculations, Eqs. 8-10 require modification by introducing separate energy equations for each phase. The model we use is an extension of that in [11], without some of its simplifying assumptions (in particular, we do not assume that the diffusion of heat in the gas phase is negligible):

$\delta\left(\frac{\partial}{\partial t}\left(\rho_{\mathrm{g}} \phi T_{\mathrm{g}}\right)+\frac{\partial}{\partial x}\left(\rho_{\mathrm{g}} u_{\mathrm{g}} \phi T_{\mathrm{g}}\right)\right)=\frac{\gamma}{\alpha} \frac{\partial}{\partial x}\left(\phi \frac{\partial T_{\mathrm{g}}}{\partial x}\right)+\left((1-\epsilon) \Omega+\mu \alpha^{-1}\right)\left(T_{\mathrm{s}}-T_{\mathrm{g}}\right)$ 

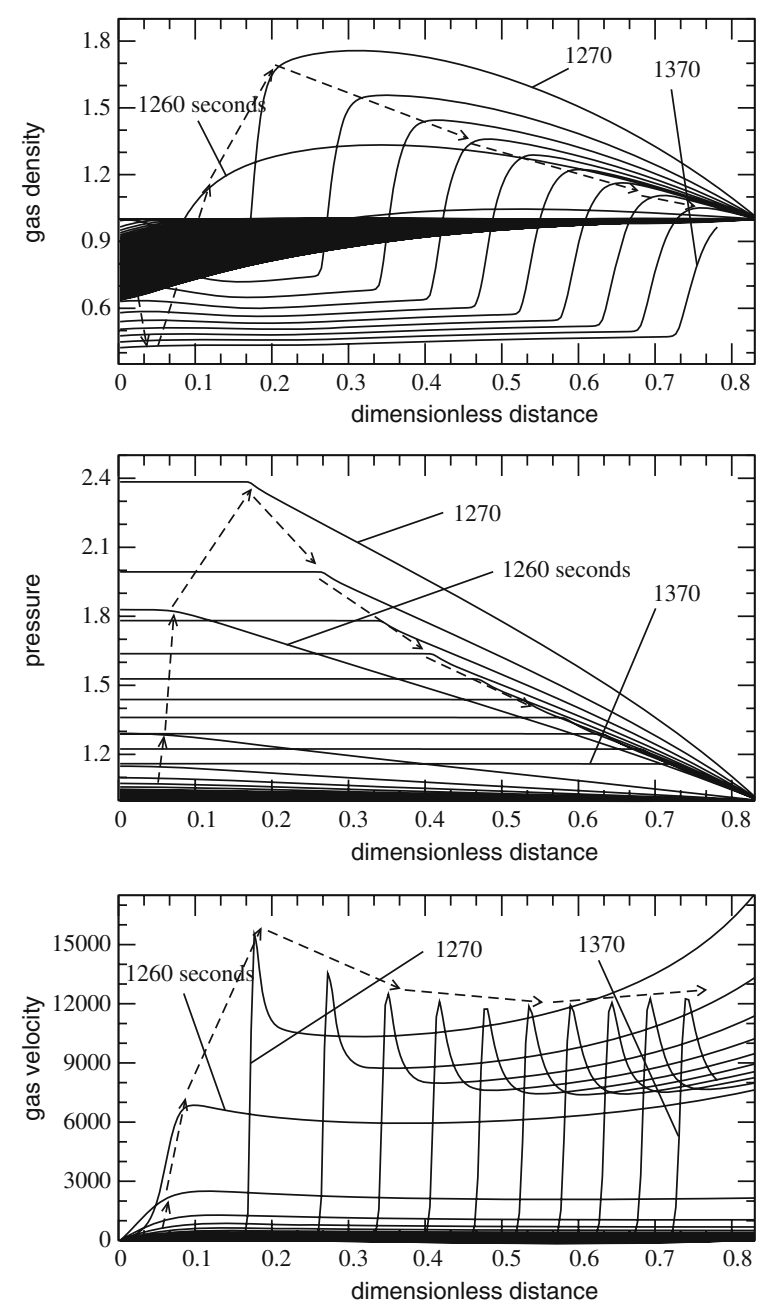

Fig. 3 Gas density, pressure and velocity profiles corresponding to Fig. 2. Note that the gas density is initially decreasing. Once a combustion wave is formed, the density ahead of the wave is much larger than behind it, where the gas is almost motionless. The arrows indicate the direction of movement of the profiles

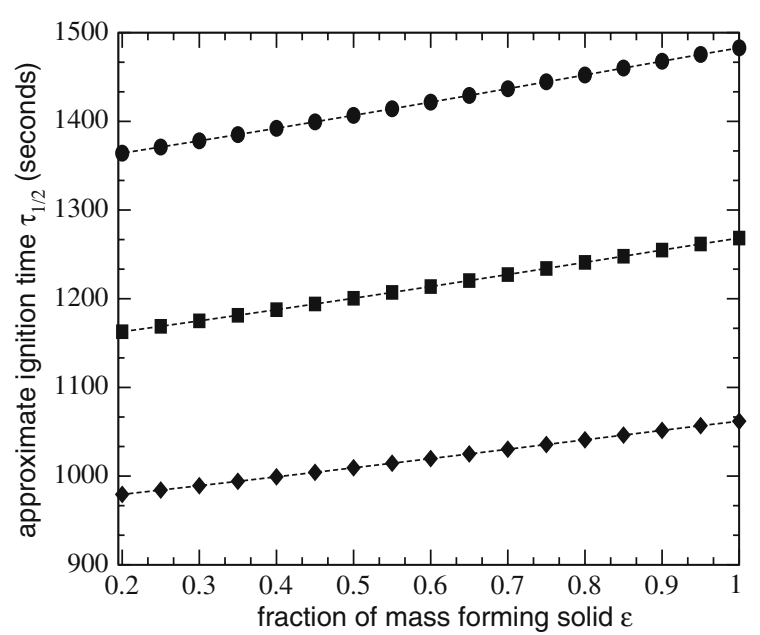

Fig. 4 Approximate ignition time, $\tau_{1 / 2}$, as a function of the fraction of reactant that forms solid, $\epsilon$. The circles indicate values for $\phi_{0}=0.2$, the squares for $\phi_{0}=0.3$ and diamonds for $\phi_{0}=0.4$

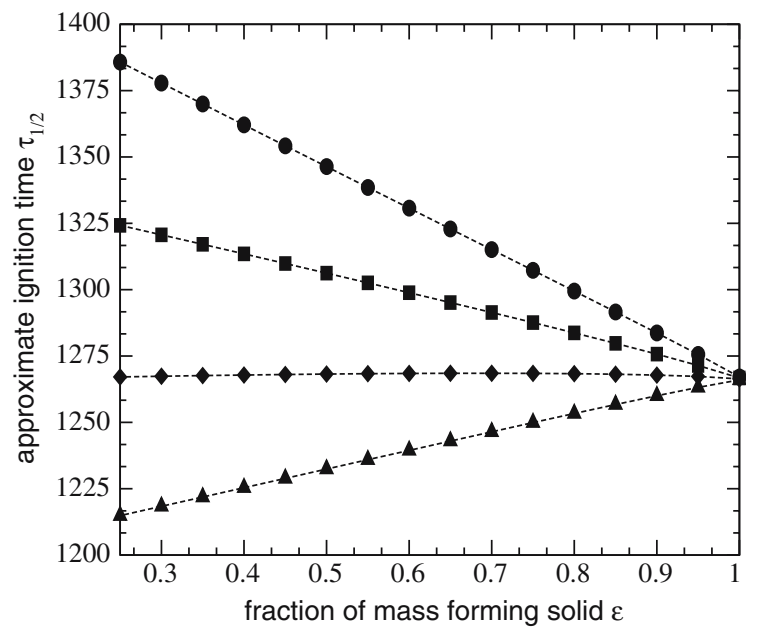

Fig. 5 Approximate ignition time, $\tau_{1 / 2}$, as a function of the fraction of reactant that forms solid, $\epsilon$, for $C_{\mathrm{g}}^{\prime}=1200 \mathrm{~J} \mathrm{~kg}^{-1} \mathrm{~K}^{-1} \quad$ (triangles); $\quad C_{\mathrm{g}}^{\prime}=1400 \mathrm{~J} \mathrm{~kg}^{-1} \mathrm{~K}^{-1}$ (diamonds); $C_{\mathrm{g}}^{\prime}=1600 \mathrm{~J} \mathrm{~kg}^{-1} \mathrm{~K}^{-1} \quad$ (squares); $C_{\mathrm{g}}^{\prime}=1800$ $\mathrm{J} \mathrm{kg}^{-1} \mathrm{~K}^{-1}$ (circles); all with $C_{\mathrm{s}}^{\prime}=1200 \mathrm{~J} \mathrm{~kg}^{-1} \mathrm{~K}^{-1}$

$$
\begin{aligned}
& \frac{\partial}{\partial t}\left((1-\phi) T_{\mathrm{s}}\right)=\frac{\partial}{\partial x}\left((1-\phi) \frac{\partial T_{\mathrm{s}}}{\partial x}\right)+Q \Omega-\mu\left(T_{\mathrm{s}}-T_{\mathrm{g}}\right) \\
& \frac{\partial}{\partial t}\left(\rho_{\mathrm{g}} \phi\right)+\frac{\partial}{\partial x}\left(\rho_{\mathrm{g}} u_{\mathrm{g}} \phi\right)=(1-\epsilon) \delta^{-1} \Omega \\
& u_{\mathrm{g}}=-k \frac{\phi^{3}}{(1-\phi)^{2}} \frac{\partial P_{\mathrm{g}}}{\partial x}
\end{aligned}
$$


$P_{\mathrm{g}}=\rho_{\mathrm{g}} T$,

$-\frac{\partial \psi}{\partial t}=\Omega \equiv A \psi \mathrm{e}^{-E / T_{\mathrm{s}}}$

$\psi=1-\frac{\phi-\epsilon \phi_{0}}{1-\epsilon}$

with boundary conditions:

$$
\begin{aligned}
& T_{\mathrm{S}}\left(x_{1}, t\right)=T_{\mathrm{g}}\left(x_{1}, t\right)=1, \quad(1-\phi) \frac{\partial T_{\mathrm{s}}}{\partial x}(0, t)+\gamma \phi \frac{\partial T_{\mathrm{g}}}{\partial x}(0, t)=-1, \\
& T_{\mathrm{S}}(0, t)=T_{\mathrm{g}}(0, t), \quad \rho_{\mathrm{g}}\left(x_{1}, t\right)=1, \quad u_{\mathrm{g}}(0, t)=0,
\end{aligned}
$$

and initial conditions:

$$
T_{\mathrm{g}}(x, 0)=T_{\mathrm{S}}(x, 0)=1, \quad \rho_{\mathrm{g}}(x, 0)=1, \quad \phi(x, 0)=1-\psi(x, 0)=\phi_{0}, \quad u_{\mathrm{g}}(x, 0)=0 .
$$

It can be shown that these equations reduce to the $\mathrm{PH}$ model as $\mu^{\prime} \rightarrow \infty$ (to see this we can eliminate the heat-exchange terms and set $T_{\mathrm{g}}=T_{\mathrm{s}}$ ).

Equations (11)-(13) have been non-dimensionalized using (7), with additionally:

$T_{\mathrm{s}}=\frac{T_{\mathrm{s}}^{\prime}}{T_{0}^{\prime}}, \quad T_{\mathrm{g}}=\frac{T_{\mathrm{g}}^{\prime}}{T_{0}^{\prime}}, \quad \mu=\frac{\mu^{\prime} \lambda_{\mathrm{s}}^{\prime} T_{0}^{\prime 2}}{p^{\prime 2}}, \quad \alpha=\frac{C_{\mathrm{g}}^{\prime}}{C_{\mathrm{s}}^{\prime}}, \quad \delta=\frac{\rho_{\mathrm{g}}^{\prime}}{\rho_{\mathrm{s}}^{\prime}}, \quad \gamma=\frac{\lambda_{\mathrm{g}}^{\prime}}{\lambda_{\mathrm{s}}^{\prime}}$.

We have repeated the last three of (7) for convenience. It is important to note the following assumptions and comments about the model (11)-(14) (also see [11]):

- Energy balances for each phase will account for heat flux through a given volume as well as heat exchange between the two phases. As mentioned above, we have assumed a linear rate of heat exchange between the solid and gas phases, with coefficient $\mu^{\prime}$, in units of $\mathrm{J} \mathrm{m}^{-3} \mathrm{~K}^{-1} \mathrm{~s}^{-1}$. Assuming that the heat is produced as solid-state reaction, we see that the energy released in the reaction is primarily spent on heating the solid. As a result, we account for heat production by reaction only in the solid energy (temperature) equation, (11b).

- We must take account of the fact that gas is produced by reaction at the surface of the solid particles, and here the solid temperature may be different from the mean gas-phase temperature. The resulting increase in heat content in the gas phase is accounted for by an extra source term on the right-hand side of the gas energy (temperature) equation, (11a). The factor $1-\epsilon$ represents the mass of gas produced per unit mass of solid reactant lost.

- We assume that the material of the hotspot is such that its surface temperature at any time is uniform (this is certainly plausible for metallic materials) and will equalize between solid contact and gas contact very rapidly, for a typical average pore size. Thus, it is realistic to assign equal gas and solidphase temperatures at the hotspot surface and to average the flux with respect to porosity (to take account of the difference in thermal conductivity between the two phases); see the first two conditions in (12).

The approximation of the ignition time used in the previous section, $\tau_{1 / 2}$, is also employed in the present section. Let us first compare the results of the $\mathrm{H}$ model using the values of Table 1, given by Figs. 6 and 7 , with those of the equivalent PH model and the system without gas flow (Figs. 1 and 2, respectively). Examination of the former reveals that the ignition time, again measured by $\tau_{1 / 2}$, is slightly shorter than that of the PH model, about $1220 \mathrm{~s}$ compared with 1240 s. Figure 8 shows the evolution of the heat exchange, $\mu\left(T_{\mathrm{g}}-T_{\mathrm{s}}\right)$, for the values given in Table 1 (corresponding to Figs. 6 and 7). In the early stages of reaction the gas temperature exceeds that of the solid in the entire region, but as the reaction proceeds the solid temperature rises more rapidly near the hotspot boundary. Eventually, it rises above the gas temperature 

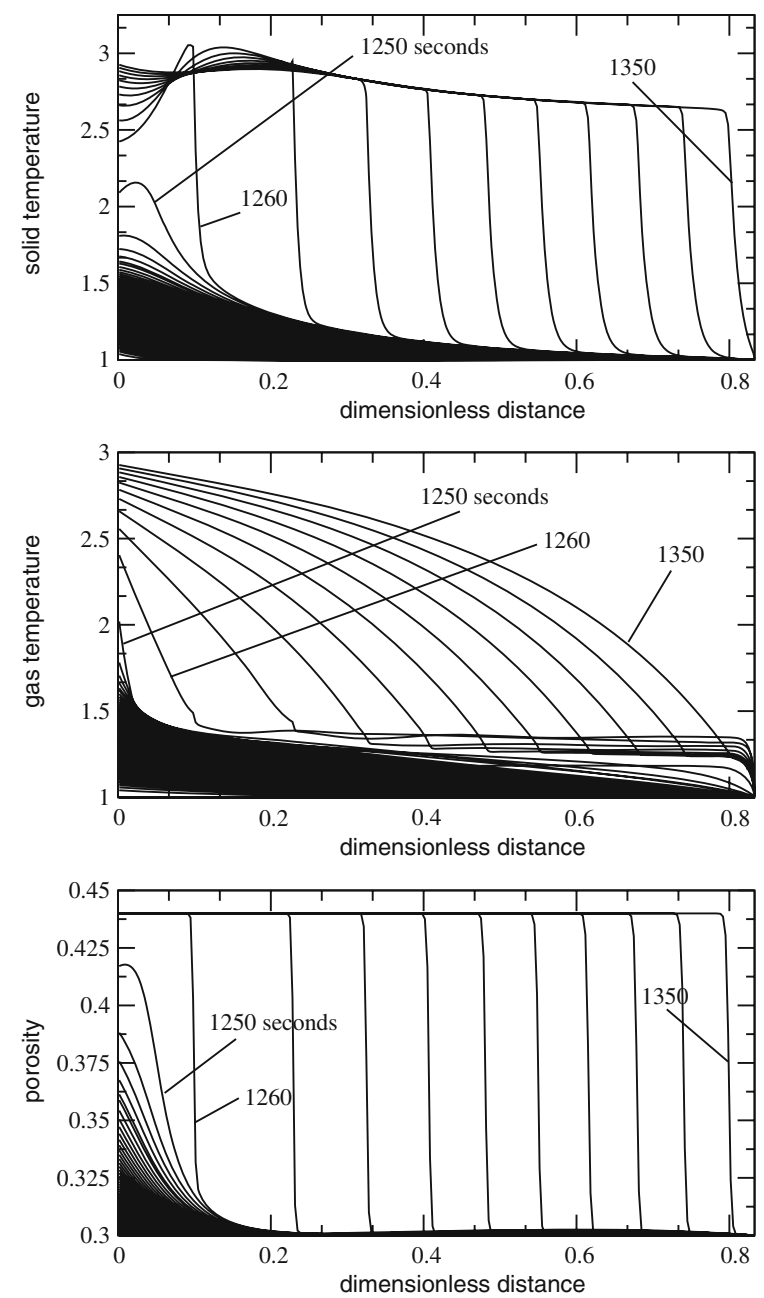

Fig. 6 Profiles of solid- and gas-phase temperature and porosity for the $\mathrm{H}$ model, using the values in Table 1. Compare the time of ignition with those of (i) no gas motion, Figure 1; and (ii) PH, Fig. 2
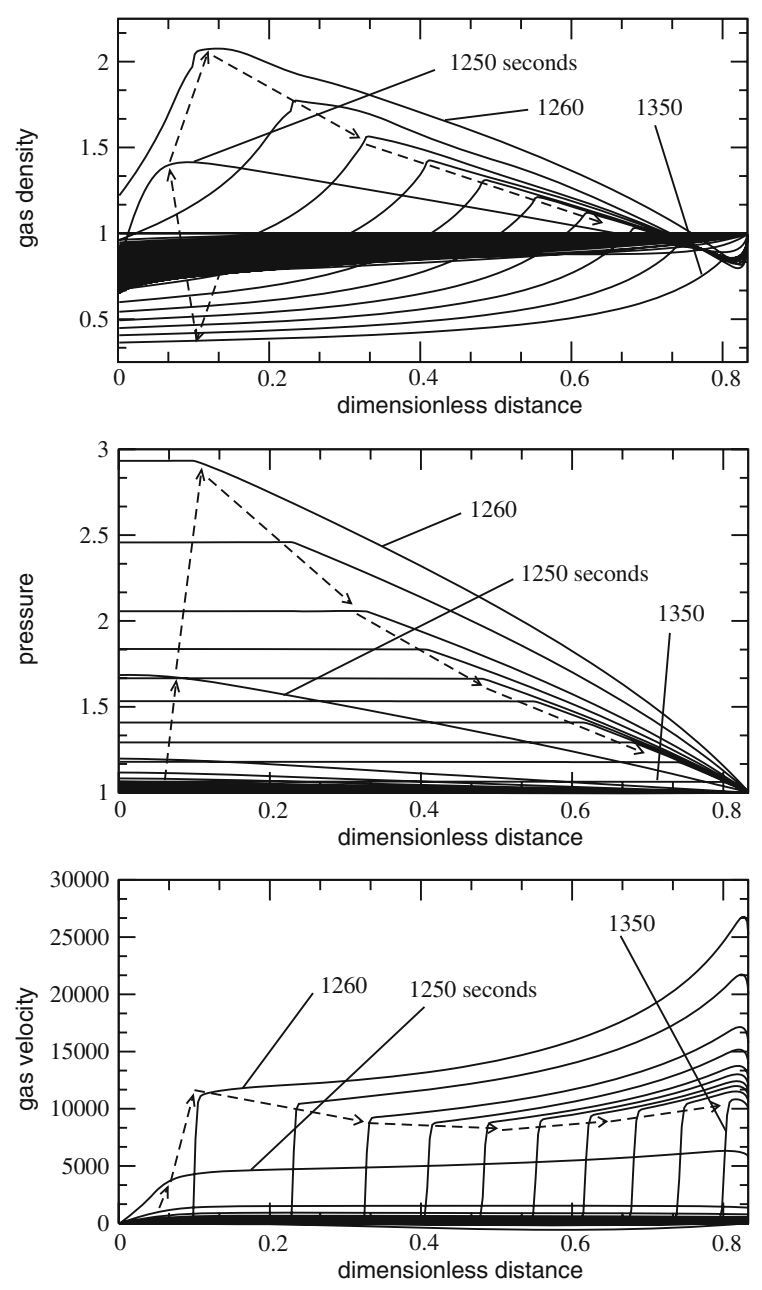

Fig. 7 Gas density, pressure and velocity profiles corresponding to Fig. 6. Note that the gas density is initially decreasing, and begins to rise when reaction begins (see caption in Fig. 3). The arrows indicate the direction of movement of the profiles

in the vicinity of the reaction zone and a sharp interface develops behind which the solid temperature dominates and ahead of which the gas temperature dominates. This interface is of course the reaction zone. Thus, ahead of the reaction zone the solid receives heat from the gas, thereby supplementing the preheating effect of conduction. Because the local temperature difference changes sign during the process just described, it is difficult to ascertain the precise effect of heat exchange on the ignition time; there is a period of gas exchanging heat to the solid and a period in which the opposite occurs. The difference in ignition times between the examples represented by Figs. 2 and 6 suggests that the period of gas exchanging heat to the solid has a weak effect in shortening the ignition time.

Figure 9 shows the evolution of the values of $T_{\mathrm{s}}, \phi, P$ and $\rho_{\mathrm{g}}$ at the hotspot, $x=x_{0}$. Note that by the boundary condition (12), $T_{\mathrm{s}}(0, t)=T_{\mathrm{g}}(0, t)$. The points represented by an asterisk highlight the value of each quantity at the time $\tau_{1 / 2}$. As can be seen by the rapid variation in each quantity soon after $\tau_{1 / 2}$, the latter represents a good indicator of the ignition time. It should be pointed out that these plots are typical. 


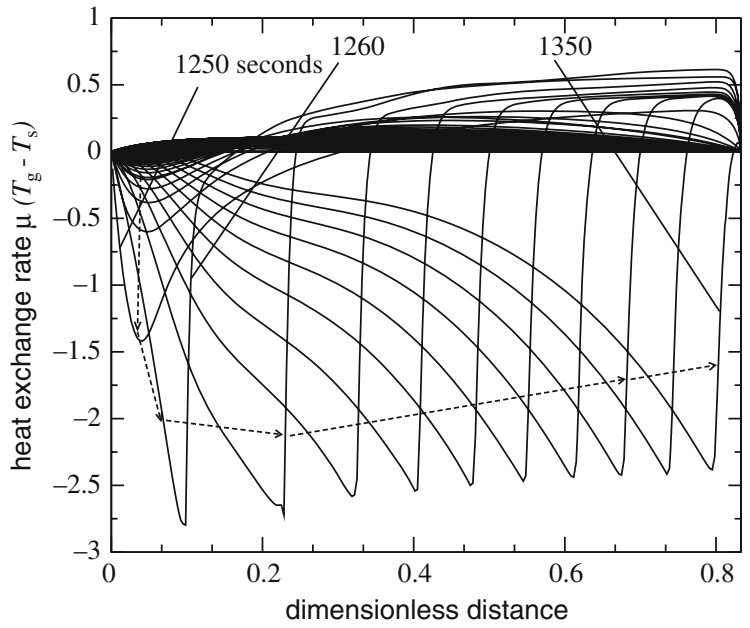

Fig. 8 Profiles of the heat exchange rate, $\mu\left(T_{\mathrm{g}}-T_{\mathrm{s}}\right)$ for $\mu^{\prime}=50 \mathrm{~J} \mathrm{~m}^{-3} \mathrm{~K}^{-1} \mathrm{~s}^{-1}$; corresponding to Figs. 6 and 7. Other values are as in Table 1 . The arrows indicate the direction of movement of the profiles.
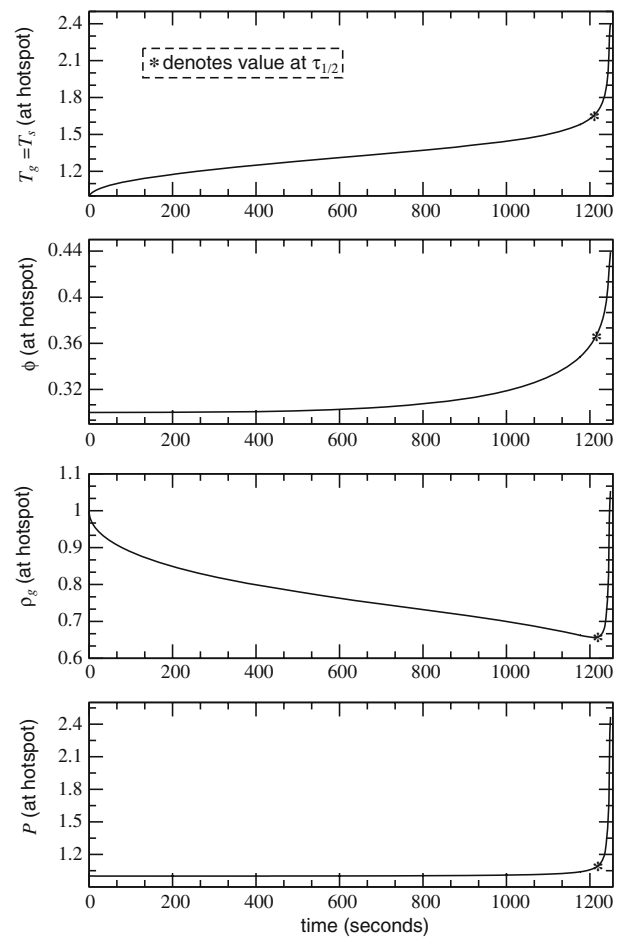

Fig. 9 Evolution of the values of $T_{\mathrm{s}}, \phi, P$ and $\rho_{\mathrm{g}}$ at the hotspot, $x=x_{0}$. Note that $T_{\mathrm{S}}(0, t)=T_{\mathrm{g}}(0, t)$. The points represented by $*$ highlight the value of each quantity at the time $\tau_{1 / 2}$. As can be seen by the rapid variation in each quantity soon after $\tau_{1 / 2}$, the latter represents a good indicator of the ignition time

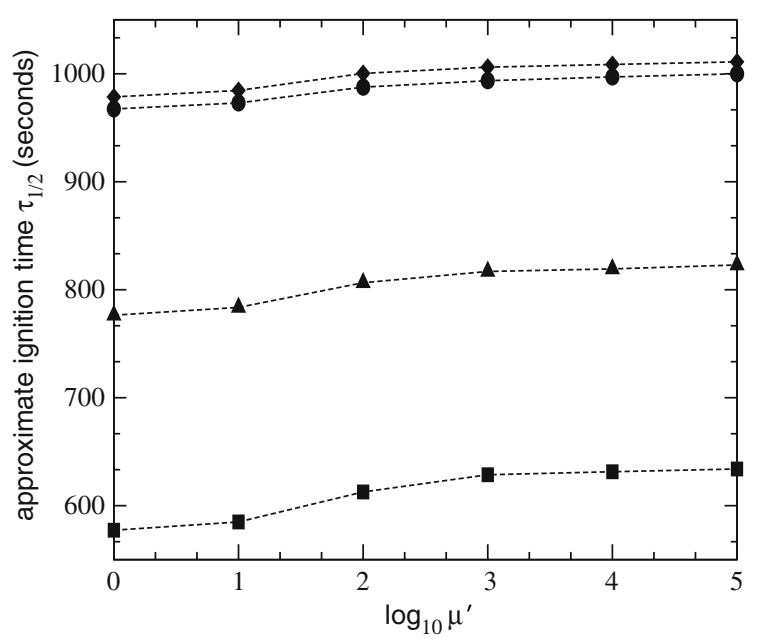

Fig. 10 Approximate ignition time $\tau_{1 / 2}$ as a function of the heat-exchange parameter $\mu^{\prime}$. The diamonds indicate values for $\phi_{0}=0.4$ and $\epsilon=0.6$; the circles for $\phi_{0}=0.4$ and $\epsilon=0.5$; the triangles for $\phi_{0}=0.5$ and $\epsilon=0.6$; and the squares for $\phi_{0}=0.6$ and $\epsilon=0 \cdot 5$. Other parameters are as in Table 1

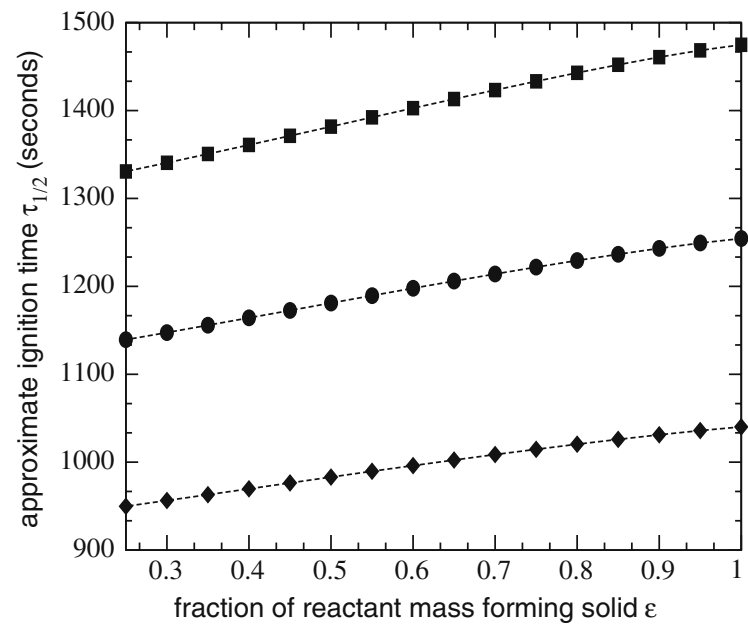

Fig. 11 Approximate ignition time $\tau_{1 / 2}$ as a function of the fraction of reactant mass that forms solid, $\epsilon$, with other parameters as in Table 1 . The circles indicate values for $\phi_{0}=0 \cdot 2$, squares for $\phi_{0}=0.3$ and triangles for $\phi_{0}=0.4$ 


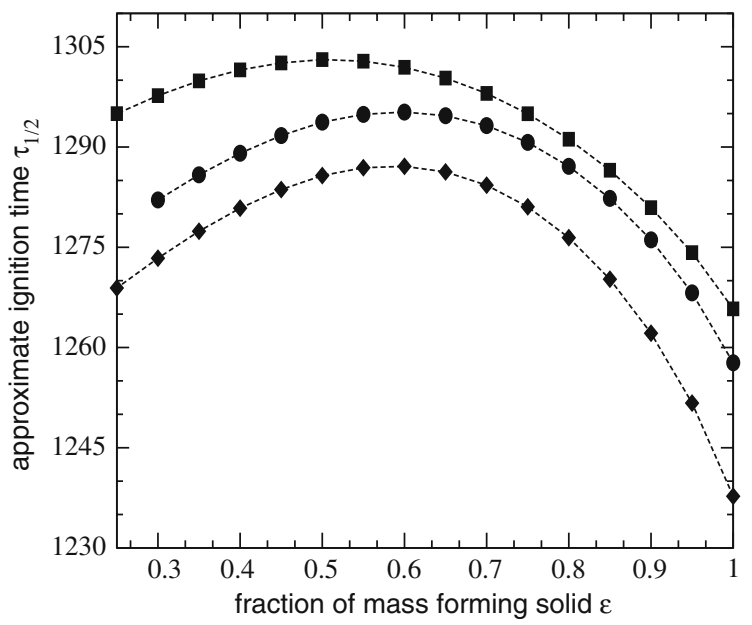

Fig. 12 Approximate ignition time $\tau_{1 / 2}$ as a function of the fraction of reactant mass that forms solid, $\epsilon$ when $C_{\mathrm{g}}^{\prime}=1800 \mathrm{~J} \mathrm{~kg}^{-1} \mathrm{~K}^{-1}$ and $C_{\mathrm{s}}^{\prime}=1200 \mathrm{~J} \mathrm{~kg}^{-1} \mathrm{~K}^{-1}$; $\mu^{\prime}=250 \mathrm{~J} \mathrm{~m}^{-3} \mathrm{~K}^{-1} \mathrm{~s}^{-1} \quad$ (squares); $\mu^{\prime}=50 \mathrm{~J} \mathrm{~m}^{-3} \mathrm{~K}^{-1} \mathrm{~s}^{-1}$ (circles); $\mu^{\prime}=1 \mathrm{~J} \mathrm{~m}^{-3} \mathrm{~K}^{-1} \mathrm{~s}^{-1}$ (triangles). Other parameters as in Table 1

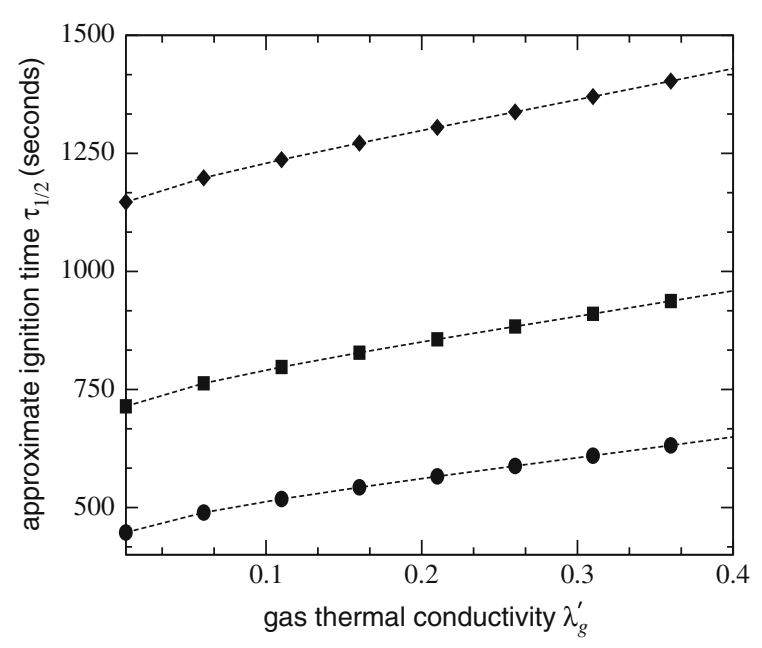

Fig. 13 Approximate ignition time $\tau_{1 / 2}$ as a function of the gas thermal conductivity, $\lambda_{\mathrm{g}}^{\prime}$. The circles indicate values for $\lambda_{\mathrm{s}}^{\prime}=0.4$, the triangles for $\lambda_{\mathrm{s}}^{\prime}=0 \cdot 2$, and the squares for $\lambda_{\mathrm{s}}^{\prime}=0 \cdot 1$. Other parameters are as in Table 1
Fig. 14 Approximate ignition time $\tau_{1 / 2}$ as a function of the solid specific heat capacity, $C_{\mathrm{s}}^{\prime}$ (other parameters values are as in Table 1). Values of $\tau_{1 / 2}$ are also given for the equivalent $\mathrm{PH}$ systems. The dashed lines correspond to

$C_{\mathrm{g}}^{\prime}=1250 \mathrm{~J} \mathrm{~kg}^{-1} \mathrm{~K}^{-1}$, the solid line to $C_{\mathrm{g}}^{\prime}=1000 \mathrm{~J} \mathrm{~kg}^{-1} \mathrm{~K}^{-1}$ and the dotted lines to $C_{\mathrm{g}}^{\prime}=750 \mathrm{~J} \mathrm{~kg}^{-1} \mathrm{~K}^{-1}$.

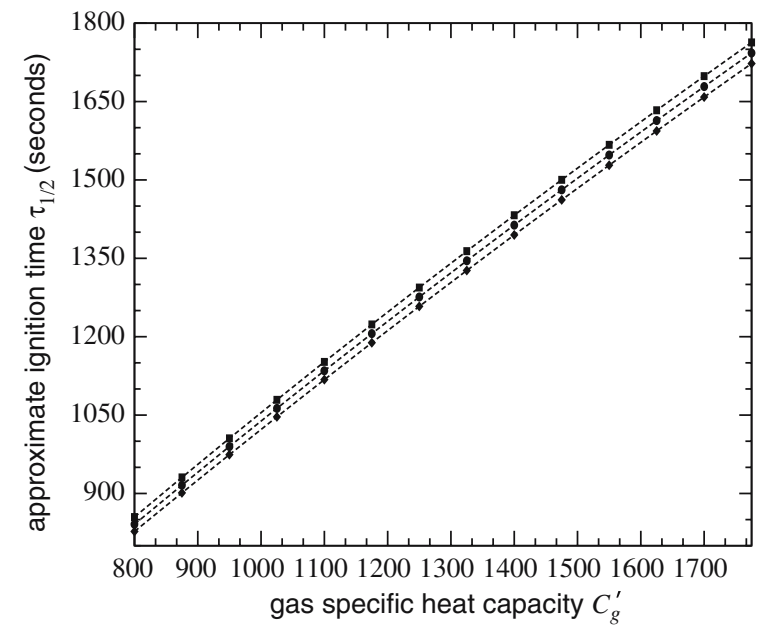

We now refer to Fig. 10 for the dependence of $\tau_{1 / 2}$ on $\mu^{\prime}$, as it is increased from $1 \mathrm{~J} \mathrm{~m}^{-3} \mathrm{~K}^{-1} \mathrm{~s}^{-1}$ to a value that corresponds to equal temperature in the gas and solid phases (the right-hand axis represents values of $\tau_{1 / 2}$ for the PH model). This figure confirms that the dependence on $\mu^{\prime}$ is relatively weak, with variations at the most of the order of $10 \%$, and then only when the volume of gas exceeds that of the solid.

Figure 11 demonstrates the change in $\tau_{1 / 2}$ as $\epsilon$ is varied, for three values of $\phi_{0}$. As with the PH model we find that decreasing $\epsilon$ decreases $\tau_{1 / 2}$, which reflects the fact that the solid phase has a much larger heat capacity than the gas, and therefore attains a lower temperature if more is present per unit volume. However, when we increase $C_{\mathrm{g}}^{\prime}$ above the value of $C_{\mathrm{s}}^{\prime}$ we find a different behaviour, as was the case for the PH model. Referring to Fig. 12 we see that the trend above is reversed for $\epsilon$ greater than approximately 0.5 , i.e., ignition time increases with deceasing $\epsilon$, but that for $\epsilon$ below approximately 0.5 ignition time decreases with decreasing $\epsilon$. As $\mu^{\prime} \rightarrow \infty$, the trend becomes linear and approaches that of the PH model; see Fig. 5. 
Specific heat capacity and thermal conductivity of the two phases play a major role, as has already been seen. Figure 13 demonstrates the dependence of $\tau_{1 / 2}$ on $\lambda_{\mathrm{g}}^{\prime}$, for three values of $\lambda_{\mathrm{s}}^{\prime}$. As $\lambda_{\mathrm{s}}^{\prime}$ is decreased, the value of $\tau_{1 / 2}$ decreases, reflecting the fact that less heat is conducted away from the reaction zone. As $\lambda_{\mathrm{g}}^{\prime}$ is increased, $\tau_{1 / 2}$ increases because the gas phase transports more heat away from the reaction zone, where its temperature decreases, implying that the solid receives less heat from the gas phase, per unit time. Note also that varying $\lambda_{\mathrm{s}}^{\prime}$ has a more significant impact on the ignition time than varying $\lambda_{\mathrm{g}}^{\prime}$.

Figure 14 shows the value of $\tau_{1 / 2}$ as $C_{\mathrm{s}}^{\prime}$ is increased, for three values of $C_{\mathrm{g}}^{\prime}$. An increase in $C_{\mathrm{s}}^{\prime}$ leads to a decrease in $\tau_{1 / 2}$, again reflecting the fact that the solid absorbs more energy and therefore attains a lower temperature. Increases in $C_{\mathrm{g}}^{\prime}$ have only a minor effect.

\section{Discussion and conclusions}

The aim of this paper has been to further the understanding of ignition in reactive porous solids subjected to heating from an impermeable hotspot. We are concerned particularly with those of a low exothermicity, which often pose storage and handling problems across a number of industries. More specifically, we have been interested in the role played by (i) the presence of gas in the pores; (ii) gas production and convection; (iii) (a finite rate of) heat exchange between the phases; and (iv) heat conduction and heat capacity of the two phases. The many, and often competing, effects in these systems (heat generation, reactant consumption, gas transport and heat-exchange) do not mean that it is a simple matter to predict their behaviour. We summarize our findings as follows:

(1) Numerical experiments without accounting for heat exchange (the PH model) reveal that for lowexothermicity materials the presence of gas can shorten the time to ignition, defined as the birth of a self-propagating combustion wave (recall Figs. 1,2,4). This effect is due primarily to the much lower heat capacity of the gas phase $\left(\rho_{\mathrm{g}}^{\prime} C_{\mathrm{g}}^{\prime}\right)$, and therefore the higher temperatures it attains, compared with the solid. However, for large enough values of gas-phase specific heat capacity (greater than that of the solid phase) ignition time increases as more gas is produced.

(2) In our numerical examples the wave speed appears to be significantly increased by the production and movement of hot gas through the porous solid, which supplements the conductive preheating by both convection and the greater diffusivity of the gas. This, and other aspects of the wave propagation have been rigorously investigated in [11].

(3) The single temperature and dual temperature models give different predictions of the ignition time, with equality only in the limit $\mu^{\prime} \rightarrow \infty$ (infinite rate of heat exchange). However, altering the rate of heat exchange between the solid and gas phases leads to only mild changes in the ignition time.

(4) As the volume of gas produced during reaction is increased, the ignition time decreases, provided the gas-phase specific heat capacity does not exceed that of the solid phase. If it does, the dependence is non-linear, having a maximum near $\epsilon=0.5$.

(5) Increasing the thermal conductivity of the gas and/or solid phase can significantly increase the ignition time.

(6) Increasing the specific heat capacity of the solid can dramatically increase the ignition time, provided it exceeds the specific heat capacity of the gas.

Overall, it appears that the presence of the gas plays an important role in determining the ignition time of the system. The rate at which it transports heat away from the reaction zone, its smaller heat capacity, and the rate at which it exchanges heat with the solid are all competing processes. Each in isolation has a considerable influence on the ignition time, the most important being the thermal conductivities of gas and, especially, solid, the specific heat capacity of the solid and the initial porosity of the material. From the safety perspective, the greater risk of initiating self-propagating, and possibly disastrous, combustion is associated with large porosity, low solid heat capacity and poor solid thermal conductivity. In general ignition is delayed for large $\epsilon$. The slight exception occurs for the (unusual) case of $C_{\mathrm{g}}^{\prime}>C_{\mathrm{s}}^{\prime}$. 


\subsection{Future directions}

Throughout these investigations we have assumed that the reaction is not oxygen-limited (which is true for some chemical compounds) and the physical characteristics of the solid product are identical to those of the reactant solid. Current work, in both one and three dimensions, has extended the model to include a gaseous oxidant component; the effects are significant and in some ways counter-intuitive. Solid materials that produce, on reaction, a solid product with different physical properties, for example a greater volume or heat capacity, or smaller thermal conductivity, could have great value in preventing the onset of unwanted self-propagating combustion, and this is the subject of ongoing modelling work. This work also includes multi-stage kinetics to capture, for example, char oxidation.

Acknowledgements The authors are grateful to the EPSRC for financial support through Research Grant GR/R 22179. We would also like to thank the referees for their helpful comments and suggestions.

\section{Appendix 1: Nomenclature}

\section{Roman}

\section{Quantity}

Pre-exponential factor

Specific heat capacity

Activation energy

Kozeny-Carmen constant

Power

Pressure

Heat of reaction

Universal gas constant

Time

Temperature

Velocity

Molar mass

Length

Greek

\section{Quantity}

Ratio of specific heats

Ratio of thermal

conductivities

Ratio of densities

Fraction of reacted mass

forming solid

Permeability

Thermal conductivity

Heat-exchange

parameter

Dynamic viscosity

Density

$\begin{array}{ll}\text { Symbol } & \text { Units } \\ A & \mathrm{~s}^{-1} \\ C & \mathrm{~J} \mathrm{~kg}^{-1} \mathrm{~K}^{-1} \\ E & \mathrm{~J} \mathrm{kmol}^{-1} \\ k & \mathrm{~kg} \mathrm{~m}^{2} \mathrm{~s} \\ p & \mathrm{~W} \mathrm{~m}^{-2} \\ P & \mathrm{~atm}^{-1} \\ Q & \mathrm{~J} \mathrm{~kg}^{-1} \\ R & \mathrm{~J} \mathrm{~mol}^{-1} \mathrm{~K}^{-1} \\ t & \mathrm{~s} \\ T & \mathrm{~K} \\ u & \mathrm{~m} \mathrm{~s}{ }^{-1} \\ W & \mathrm{~kg} \mathrm{kmol}^{-1} \\ x & \mathrm{~m}\end{array}$

$\begin{array}{ll}\text { Symbol } & \text { Units } \\ \alpha & - \\ \gamma & - \\ \delta & - \\ \epsilon & - \\ \kappa & \text { Henry m}^{-1} \\ \lambda & \mathrm{W} \mathrm{m}^{-1} \mathrm{~K}^{-1} \\ \mu & \mathrm{J} \mathrm{m}^{-3} \mathrm{~K}^{-1} \mathrm{~s}^{-1} \\ & \\ \nu & \mathrm{Pa} \mathrm{s}^{-3} \\ \rho & \mathrm{kg} \mathrm{m}^{-3}\end{array}$




$\begin{array}{ll}\begin{array}{ll}\text { Porosity } \\ \text { Reactant volume } \\ \text { fraction }\end{array} & \phi \quad- \\ \text { Reaction rate } & \Omega \quad \mathrm{kg} \mathrm{m}^{-3} \mathrm{~s}^{-1} \\ \text { Subscripts } & \\ \text { Symbol } & \text { Meaning } \\ g & \text { Gas property } \\ s & \text { Solid property } \\ 0 & \text { Ambient/initial value }\end{array}$

Superscripts

Symbol

Meaning

dimensional quantity

\section{Appendix 2: Numerical method}

The systems (2)-(6) and (11)-(13) were solved using the finite-volume method, with first-order centraldifferencing for the diffusion terms and first-order downwind-differencing for the convection terms. The grid was staggered with respect to the gas velocity. The first-order time-stepping was made semi-implicit by approximating the linear terms by their values at the advanced time and the non-linear terms by their values at the current time and iterating. Convergence to within a good degree of accuracy was typically achieved with only a few (less than 10) iterations. As an example, we demonstrate the procedure for the temperature equation (8a), in the SPT model. First we write it in a more convenient form:

$$
\left(1-\phi+\alpha \delta \rho_{\mathrm{g}} \phi\right) \frac{\partial T}{\partial t}+\alpha \delta \frac{\partial}{\partial t}\left(\rho_{\mathrm{g}} \phi\right)+\alpha \delta \frac{\partial}{\partial x}\left(\rho_{\mathrm{g}} u_{\mathrm{g}} \phi T\right)-\frac{\partial}{\partial x}\left((1-\phi+\gamma \phi) \frac{\partial T}{\partial x}\right)=(Q+(1-\epsilon) T) \Omega
$$

using (8e) to eliminate $\partial \phi / \partial t=-(1-\epsilon) \partial \psi / \partial t=(1-\epsilon) \Omega$.

Let $\Delta x$ and $\Delta t$ be the grid-point spacing and time step, respectively; the subscript $j=1, \ldots, N$ refer to the (spatial) grid point; the superscript $t$ refer to the current time, $t+\Delta t$ to the advanced time (at which solutions are sought), and $t I$ to the intermediate solution (during the iteration procedure); then, after integrating over the domain $0 \leq x \leq x_{1}$, the discretized version of (15) is:

$$
\begin{aligned}
& \left(1-\phi_{j}^{t I}+\alpha \delta \rho_{\mathrm{g}, \mathrm{j}}^{t I} \phi_{j}^{t I}\right)\left(T_{j}^{t+\Delta t}-T_{j}^{t}\right) \frac{\Delta x}{\Delta t}+\alpha \delta T_{j}^{t+\Delta t}\left(\rho_{\mathrm{g}, \mathrm{j}}^{t I} \phi_{j}^{t I}-\rho_{\mathrm{g}, \mathrm{j}}^{t} \phi_{j}^{t}\right) \frac{\Delta x}{\Delta t} \\
& \quad+\alpha \delta\left(\rho_{\mathrm{g}, \mathrm{j}}^{t I} u_{\mathrm{g}, \mathrm{j}+1}^{t I} \phi_{j}^{t I} T_{j}^{t+\Delta t}-\rho_{\mathrm{g}, \mathrm{j}-1}^{t I} u_{\mathrm{g}, \mathrm{j}}^{t I} \phi_{j-1}^{t I} T_{j-1}^{t+\Delta t}\right)-\frac{T_{j+1}^{t+\Delta t}-2 T_{j}^{t+\Delta t}+T_{j-1}^{t+\Delta t}}{\Delta x} \\
& =\left(Q+(1-\epsilon) T_{j-1}^{t I}\right) A \psi_{j}^{t I} \exp \left(-\frac{1}{\epsilon T_{j}^{t I}}\right), \quad j=2, \ldots, N-1
\end{aligned}
$$

The endpoints $j=1$ and $j=N$ are treated slightly differently to incorporate the boundary conditions (9). Equation (8b) was treated similarly, as was Eq. (8e) after adding a negligible diffusion term for simplicity. The discretization of Eq. (8c) led to algebraic conditions, and that of Eq. (8d) was achieved by first-order backward differencing, using the intermediate (iterated) solutions in both cases.

Adaptivity in time was introduced via the following condition:

$\triangle x / \triangle t>k \times\left|u_{M}\right|$,

where $\left|u_{M}\right|$ was the maximum of the absolute value of gas velocity over $j$, and $k>0$ is a constant. It was found that a value of $k=1000$ was adequate in most cases. 


\section{References}

1. Liñan A, Williams FA (1971) Theory of ignition of a reactive solid by constant energy flux. Combust Sci Technol 3:91-98

2. Kapila AK (1981) Evolution of a deflagration in a cold combustible subjected to a uniform energy flux. Int J Eng Sci 19m:495-509

3. Telengator AM, Margolis SB, Williams FA (1999) Ignition analysis of a porous energetic material: II. Ignition at a closed heated end. Combust Theory Modell 3:433-445

4. Telengator AM, Margolis SB, Williams FA (1999) Analysis of ignition of a porous energetic material. Combust Theory Modell 3:33-49

5. Brindley J, Griffiths JF, McIntosh AC (2001) Ignition phenomenology and criteria associated with hotspots embedded in a reactive material. Chem Engng Sci 56:2037-2046

6. Brindley J, Griffiths JF, McIntosh AC (2001) Potential hazards from spherical hotspots in reactive solids. In Bradley D, Drysdale D, Makhviladze G (eds) Proceedings third international seminar fire explosion hazards, English Lake District, pp 455-463

7. Brindley J, Griffiths JF, McIntosh AC, Zhang J (1999) Initiation of combustion waves in solids and the effect of geometry. ANZIAM J 43:149-163

8. Shah A, McIntosh AC, Brindley J, Griffiths JF, Pourkashanian M (2003) The effect of oxygen starvation on ignition phenomena in a reactive solid subject to a constant heat flux. Combust Theory Modell 7:509-523

9. Shah A, Brindley J, Griffiths JF, McIntosh AC, Pourkashanian M (2004) The ignition of reactive solids by constant heating. PSEP, Trans ICheme 82:1-15

10. Aldushin AP, Seplyarskii BS (1992) Propagation of the front of an exothermic reaction through porous media under blow-through gas conditions. Soviet Phys Doklady 27:483-486

11. Wahle CW, Matkowsky BJ, Aldushin AP (2003) Effects of gas-solid non-equilibrium in filtration combustion. Combust Sci Technol 175:1389-1499

12. Seplyarskii BS (2000) Ignition of porous bodies under conditions of counterflow non-stationary filtration of a gas. Combus Explos Shock Waves 36:442-451

13. Seplyarskii BS, Gordopolova IS (1999) Ignition of porous materials by gas filtration (unsteady downstream filtration). Combus Explos Shock Waves 35:43-52

14. Seplyarskii BS (1991) Ignition of condensed systems with gas filtration. Combus Explos Shock Waves 27:1-10

15. Margolis SB (1998) Influence of pressure-driven gas permeation on the quasi-steady burning of porous energetic materials. Combust Theory Model 2:95-113

16. Margolis SB, Telengator AM (2001) Influence of sublimation and pyrolysis on quasi-steady deflagrations in confined porous energetic materials. Combust Flame 127:2135-2156

17. Margolis SB, Telengator AM, Williams FA (2002) Intrusive-limit deflagrations in confined porous energetic materials. Proc Combust Inst 29:2913-2922

18. Margolis SB, Telengator AM, Williams FA (2003) Influence of subsurface gaseous combustion on the burning of confined porous energetic materials. Combust Sci Technol 175:421-452

19. Kumar M, Kovacic SM, Kuo KK (1981) Flame propagation and combustion processes in solid propellant cracks. AIAA J 19:610-618

20. Kumar M, Kuo KK (1981) The effect of deformation on flame spreading and combustion in propellant cracks. AIAA J 19:1580-1589

21. Smirnov NN (1991) The model for combustion of porous propellants subjected to fragmentation. Combust Explos Shock Waves 27:56-63

22. Bolton EW, Lasaga AC, Rye DM (1999) Long-term flow/chemistry feedback in a porous medium with heterogeneous permeability: kinetic control of dissolution and precipitation. Am J Sci 299:1-68

23. Epstein N (1989) On the tortuosity and the tortuosity factor in flow and diffusion through porous media. Chem Engng Sci 44:777-779

24. Priestley I. Chemical Team Leader, Process Hazard Section, Syngenta. private communication 